\title{
Modeling of Hybrid Systems by Piecewise Decomposition*
}

\author{
S. Esteva, and J.L. de la Rosa \\ Universitat de Girona \\ Av. Lluis Santalo S/N, \\ 17071 Girona, Spain \\ (sesteva, peplluis) Oeia.udg.es
}

\author{
N. Rakoto-Ravalontsalama \\ Ecole des Mines de Nantes / IRCCyN \\ 4. rue Alfred Kastler \\ F-44307 Nantes Cedex 03, France \\ rakoto@emn.fr
}

\begin{abstract}
Piecewise linear models systems arise as mathematical models of systems in many practical applications, often from linearization for nonlinear systems. There are two main approaches of dealing with these systems according to their continuous or discrete-time aspects. We propose an approach which is based on the state transformation, more particularly the partition of the phase portrait in different regions where each subregion is modeled as a two-dimensional linear time invariant system. Then the Takagi-Sugeno model, which is a combination of local model is calculated. The simulation results show that the Alpha partition is well-suited for dealing with such a system.
\end{abstract}

Keywords: Hybrid systems, piecewise linear systems, Takagi-Sugeno model.

\section{Introduction}

Piecewise linear systems arise as mathematical models of systems in many practical applications, often from linearization for nonlinear systems [6] and [10]. By piecewise linear, we refer to a dynamic system that has different linear dynamics in different regions of the contimuous state space. For a historical list of references, one can refer to [6] and the references therein.

For the analysis of piecewise linear systems, the different approaches can be divided into two main classes: For discrete time dynamics, some attempts have been made to formulate analysis procedure based on properties of affine mapping and polyhedral sets [10]. For continuous time dynamics, a method for qualitative analysis of piecewise linear systems based on vector field considerations have been developed [7].

Modeling of a system using piecewise linear approximations is an interesting approach applied to nonlinear systems, in [12] the embedding theory for nonlinear systems is used in order to apply linear subspace identification, in [3] a nonlinear adaptive structure using networks of piecewise linear systems is studied and more recently in [5] a clustering technique for identification of piecewise affine systems is used for obtaining a model

\footnotetext{
"0-7803-7952-7/03/\$17.00 (C) 2003 IEEE.
}

of a hybrid system. On the other hand, Takagi-Sugeno models have been used for modeling nonlinear systems with good approximations, see [1], [9] and [2] for some approaches. The idea of partitioning the phase portrait has been proposed, amomg all other approaches, in [8].

In this paper we propose an approach that decomposes the phase portrait in different regions where each subregion is modeled as a two-dimensional linear time invariant (LTI) sy'stem. In order to complete the model defining the transition functions the TakagiSugeno framework based in fuzzy sets theory is used.

This paper is organized as follows. First the state transformation idea is explained in Section 2, in Section 3 the Takagi-Sugeno model is presented and its relation with the piecewise linear systems is defined. Section 4 presents an application to illustrate the methodology. Finally some conclusions are given in Section 5 .

\section{State Transformation}

One approach of linearization of nonlinear system is the change of coordinates. In our case the objective is to reduce the number of the local models maintaining the degree of the approximation.

Given a state vector $X=\left[x_{1}, x_{2}\right]^{T}$, the following transformation is applied:

$$
g\left(x_{1}, x_{2}\right)=\alpha=\arctan \left(\frac{\Delta x_{1}}{\Delta x_{2}}\right) .
$$

This function transforms a two-dimension vector into a scalar one. Considered with the measured output signal $y(t)$, we represent a compact representation of the internal state and the measured output, using a polar representation $[\alpha, y]$ where the bounds of each decomposed region and the corresponding local model are obtained by an identification methodology presented in [4]. This representation is called the Alpha Graph.

The state variables are obtained as a measure of the signals: the output and its variation, the last measurable or estimated by the output signal. The arc tangent function transforms $\mathcal{R}^{2}$ into $\mathcal{R}$, reducing one dimension of the state space and aggregating the output measure 


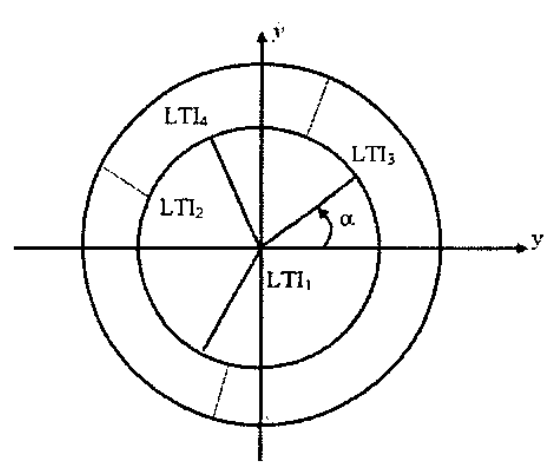

Figure 1: The Local Model Partitions

$Y(t)$ as $r$, the second component of the polar representation $[\alpha, r]$ (Figure 1), where $\alpha$ represents the dynamics and $r$ the output signal. The scaling factor $K . X i$ is eliminated by the division factor $\frac{\Delta x_{1}}{\Delta x_{2}}$ in the alpha measure. This means that the scaled signals reproduce the same dynamic expression. Decoupling the two states from the dynamics and amplitude, the alpha signal is related to the dynamics and the output to the amplitude, it is possible to partition the range of variation into the regions according the local models. Partition for the output measure, related by the non-linearities of the gain, and the partition of the alpha measure according the local linear models, from the linear or non-linear dynamics of the system depending on bilinear factors, Volterra components for example.

In this manner the controllers are decoupled, and the control action is the combination of the two signals, the control action to sequencing the local models to stabilise the system, and the control action to adjust the output signal at desired set point. The gain controller $K_{a}$ is a Gain Scheduling factor, which assigns different gain according the local model approximated, measured by the alpha limits for each region. Of the same form, $K_{y}$ is a gain factor governed by the output measure on the local limits.

The objective is to identify the local models and its bounds, where each subregion is modeled by a secondorder linear time invariant (LTI) equation.

\section{Takagi-Sugeno Model}

The Takagi-Sugeno model [11] is a combination of LTI local models. The membership functions of each LTI model is obtained with the outputs of the nonlinear signals.

The fuzzy linear model proposed by Takagi and Sugeno is a set of fuzzy rules, where the antecedent component is a fuzzy subset and its consequent component is a dynamical model, with the addition of fuzzy reasoning. The rules can be modeled as follows,

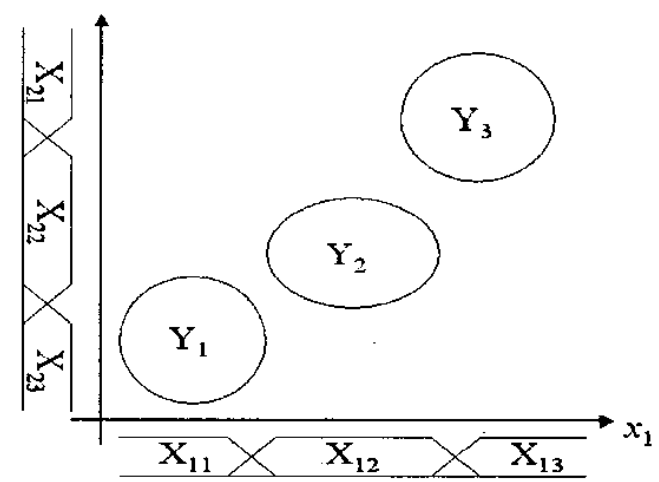

Figure 2: The Takagi-Sugeno Model

$$
\begin{aligned}
& R_{i}: \text { if } x_{1} \text { is } X_{1 i} \text { and } \ldots \text { and } x_{r} \text { is } X_{r i} \\
& \text { then } y_{i}(k)=a_{i 1} x_{1}+a_{i 2} x_{2}+\ldots+a_{i r} x_{r}
\end{aligned}
$$

The overall output of the model is computed by

$$
y=\frac{\sum_{i=1}^{L} \tau_{i} y_{i}}{\sum_{i=1}^{L} \tau_{i}}=\frac{\sum_{i=1}^{L} \tau_{i}\left(a_{i 1} x_{1}+a_{i 2} x_{2}+\ldots+a_{i r} x_{r}\right)}{\sum_{i=1}^{L} \tau_{i}}
$$

where $\tau_{i}$ is the firing strength of rule $R_{i}$, which is defined as

$$
\tau_{i}=X_{i 1}\left(x_{1}\right) \times X_{i 2}\left(x_{2}\right) \times \ldots \times X_{i r}\left(x_{r}\right)
$$

The Takagi-Sugeno Model can be viewed as a partition of the space-state where the transitions between the models are smooth, as is schematized in Figure 2.

Using the Alpha Graph, the membership functions are calculated by the variable $\alpha$ and the measured output $y(t)$, according to the partition seen previously.

In this case the partition function is used as a supervisor of the process whose determine de linear local model of the non-linear process based on the fuzzy representation and choice the appropriated regulator pre-designed for the linear model. In this form, the analysis of the controller and the system can be achieved using the supervision techniques available for hybrid systems, such as is studied in [4]. This methodology is schematized in Figure 3.

\section{Application}

The transformation method is now applied to the following system:

$$
\left\{\begin{array}{l}
\dot{x}(t)=-1.6 x_{1}(t) u(t)-3.3 x_{2}(t)-3 x_{3}(t)-x_{4}(t)+u(t) \\
\dot{x_{2}}(t)=x_{1}(t), \dot{x_{3}}(t)=x_{2}(t), \dot{x_{4}}(t)=x_{3}(t), y(t)=x_{4}(t)
\end{array}\right.
$$

This system has been simulated with MATLAB using the SIMULINK block diagrams. The simulation results show that the system can be divided into 5 different LTI 


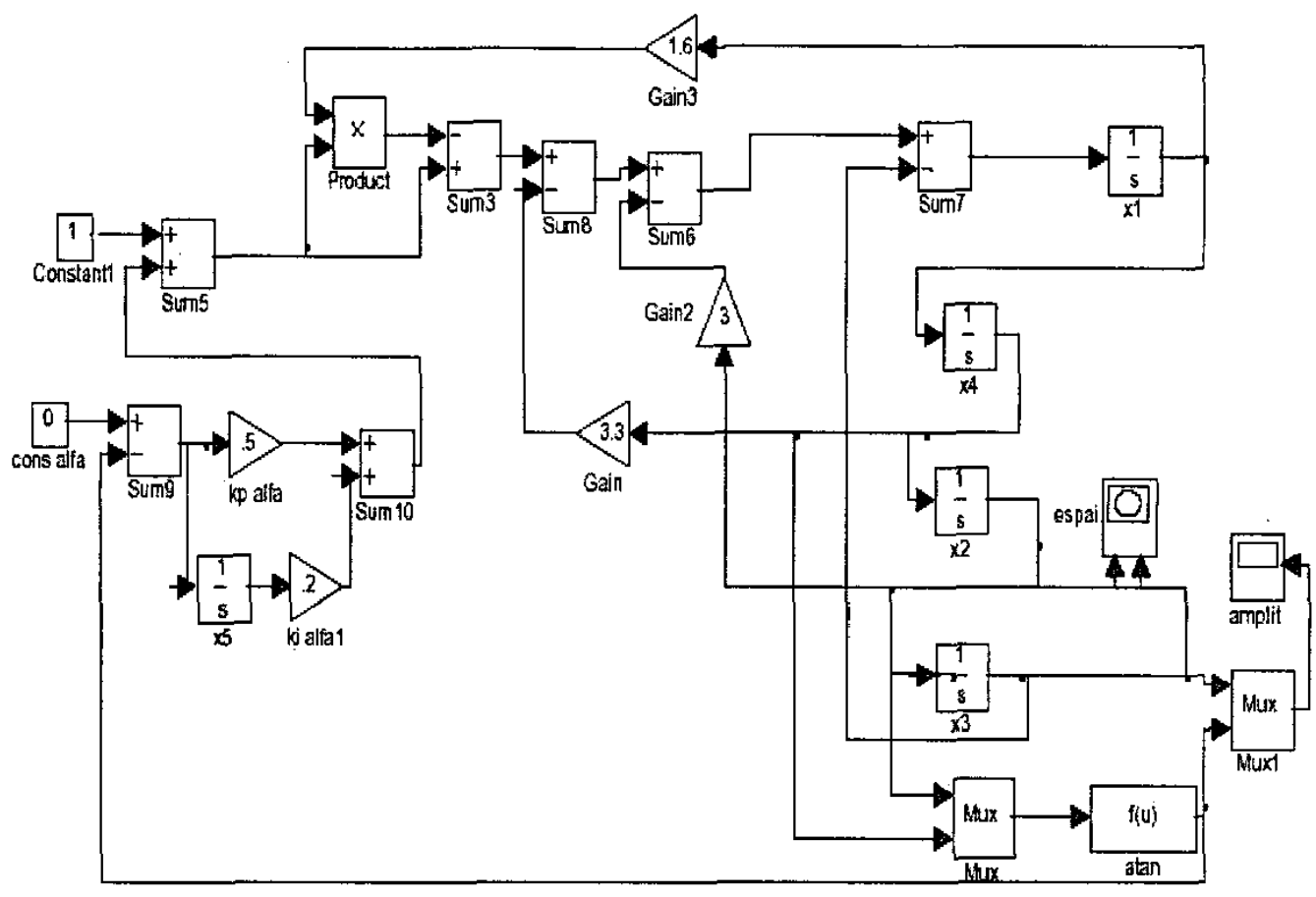

Figure 5: The MATLAB SIMULINK Model

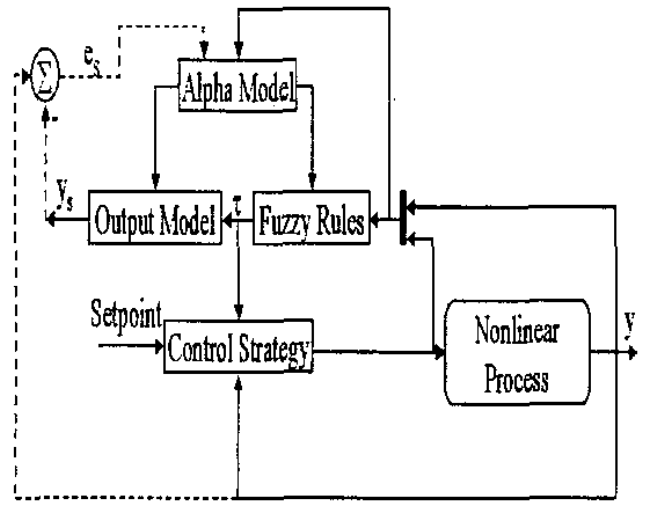

Figure 3: The Methodology

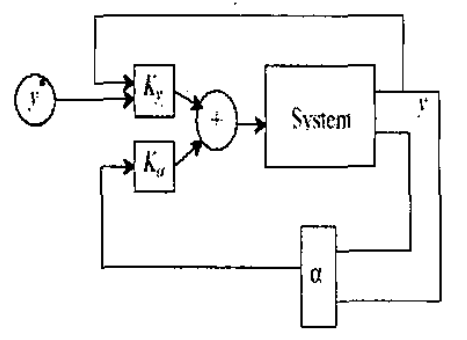

Figure 4: The Control Scheme

models, according to the value of the measured output $y(t)$. The step response shows that the signal has a small delay and the other signal is oscillating as depicted in Figure 7. In Figure 6, the output signal unstable and oscillating. A more complete set of results can be found in the $\mathrm{PhD}$ Thesis report [4].

\section{Conclusions}

We have presented in this paper a new approach for modeling a particular class of hybrid systems: The piecewise linear systems. This approach is based on the state transformation, more particularly the partition of the phase portrait in different regions where each subre- 


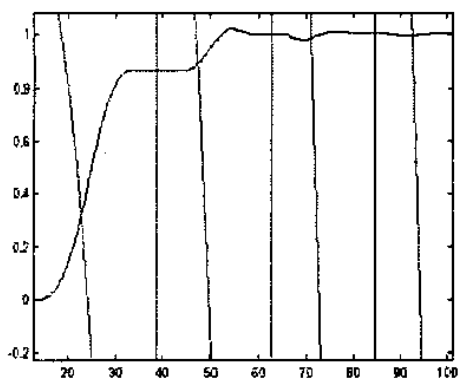

Figure 6: Some Simulation Results 1

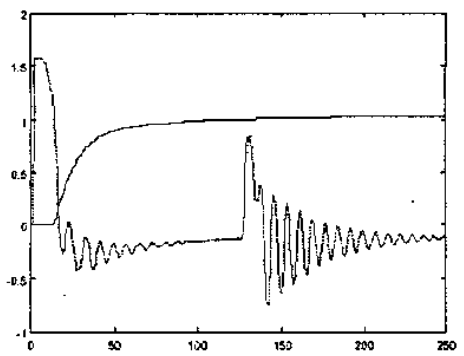

Figure 7 : Some Simulation Results 2

gion is modeled as a two-dimensional linear time invariant system. Then the Takagi-Sugeno model, which is a combination of local model is calculated. The simulation results show that the Alpha partition is well-suited for dealing with such a system.

\section{Acknowledgment}

The authors would like to thank Prof. Joseph Aguilar-Martin for some fruitful discussion about the approach presented in this paper.

\section{References}

[1] Y.H. Bang, C. K. Yoo, I.B. Lee. Nonlinear PLS Modeling with Fuzzy Inference System. Cheometrics and Intelligent Laboratory Systems 64, pp. 137 155,2003 .

[2] J.Q. Chen, Y.G. Xi, Z. J. Zhang. A Clustering Algorithm for Fuzzy Model Identification. Fuwwy Sets and Systems 98 pp. 319-329, 1998.

[3] J.Y. Choi and J. Farrell. Nonlinear Adaptive Control Using Networks of Piecewise Linear Approximations. University of California, Riverside. Technical Report NO. SNL-EE-TR-1999.18

[4] S. Esteva. "Modelling, Control and Supervision for a Class of Hybrid Systems". PhD Thesis, University of Girona, Spain, March 2003.
[5] G. Ferrari-Trecate, M. Muselli, D. Liberati, M. Morari. A Clustering Technique for the Identification of Piecewise Affine Systems. Automatica 39, pp. 205-217, 2003.

[6] M. Johansson. "Piecewise Linear Control Systems". PhD Thesis, Lund Institute of Technology, Sweden, 1999.

[7] N.B.O.L. Pettit, "Analysis of Piecewise Linear Dynamical Systems." Research Studies Press Ltd. /John Wiley and Sons Ltd., 1995.

[8] N. Rakoto-Ravalontsalama and J. Aguilar-Martin. "Automatic clustering for symbolic evaluation for dynamical system supervision" In Proc. of American Control Conference (ACC'92), vol. 3, pp. 18951897, Chicago, IL, USA, 1992.

[9] J.A. Roubos, S. Mollov, R. Babuska, H. B. Verbruggen. Fuzzy Model-Based Predictive Control Using Takagi-Sugeno Models. International Journal of Approximate Reasoning 22, pp. 3-30, 1999

[10] E. Sontag "Nonlinear Regulation: The Piecewise Linear Approach"; IEEE Trans. on Automatic Control vol. 26, pp. 346-358, 1981.

[11] T. Takagi and M. Sugeno, "Fuzzy Identification of Systems and Its Applications to Modeling and Control", IEEE Transactions on Systems, Man and Cybernetics Vol. 15, pp. 116-132, Jan 1985.

[12] V. Verdult, M. Verhaegen and J. Scherpen. Identification of Nonlinear Nonautonomous State Space Systems form Input-Output Measurements. In: Proc. of the IEEE Int. Conf. on Industrial Technology, Goa, India, pp. 410-414, January, 2000 